\title{
Evaluation of selected mechanisms of immune tolerance in psoriasis
}

\author{
Agnieszka Owczarczyk-Saczonek, Joanna Czerwińska, Małgorzata Orylska, Waldemar Placek
}

Department of Dermatology, Sexually Transmitted Diseases and Clinical Immunology, University of Warmia and Mazury, Olsztyn, Poland

\author{
Adv Dermatol Allergol 2019; XXXVI (3): 315-324 \\ DOI: https://doi.org/10.5114/ada.2019.85641
}

\begin{abstract}
Introduction: Psoriasis is an autoimmune disease with an excessively aberration of the Th17/Treg balance and deficiency of anti-inflammatory cytokines.

Aim: Evaluation of Treg markers expression in the lesional and perilesional psoriatic skin and serum anti-inflammatory cytokines in male psoriatic patients compared to healthy men.

Material and methods: Treg markers (FoxP3+, CD4, CTLA-4, CD25/IL-2R, CD39/ENTPD1, IL-7R/CD127, CD3) and tissue expression of protective cytokines (IL-10, IL-35, TGF- $\beta$ ) in the lesional and perilesional psoriatic skin from 33 male patients compared to 6 healthy skin samples were evaluated by immunohistochemistry. ELISA was used to assess serum IL-10, IL-35 and TGF- $\beta$ levels.

Results: The serum levels of IL-35, IL-10 and TGF- $\beta 1$ were higher in psoriatic patients than in controls but without any statistically significant relationship with PASI. The expressions of IL-35, CD4, IL-10, TGF- $\beta 1$, CD3, FOXP3 and CD25/IL-2R were varied in different experimental groups $(p<0.05)$. The level of IL-35 was the lowest in psoriatic lesions $(p<0.05)$ compared to perilesional skin and to controls. CD4, IL-10 and TGF- $\beta 1$ expressions were higher $(p<0.05)$ in perilesional skin than in lesions. TGF- $\beta 1$ expression was decreased in psoriatic lesions compared to controls $(p<0.05)$. CD25/IL2R expression was increased in healthy skin compared to psoriatic skin $(p<0.05)$. FOXP3 expression was elevated in psoriatic skin compared to healthy and perilesional one. There was no difference between experimental groups in CTLA-4, IL7R/CD127 and CD39/ENTPD1 expression.

Conclusions: The differences between the levels of protective cytokines and expression of Treg markers might explain the inflammation development in psoriasis.
\end{abstract}

Key words: psoriasis, Treg, interleukin 10, interleukin 35, transforming growth factor $\beta$.

\section{Introduction}

Psoriasis is a chronic inflammatory disease with gene predisposition, which can be triggered by environmental factors. Pathogenesis is characterized by an activation of Th1/Th2 axis and abnormalities of the Th17/Treg balance [1]. An essential factor of pathogenesis is the dysfunction of regulatory lymphocytes (Tregs), which are involved in homeostasis mechanisms to maintain tolerance and prevent autoimmune disorders [2].

Tregs are a heterogenous subpopulation of lymphocytes responsible for suppressing an autoreactive immune response (Table 1). They interact directly through membrane receptors for immune cells (T-lymphocytes, memory and NK, B lymphocytes, antigen presenting cells), by secretion of suppressing cytokines (IL-10, IL-35, TGF- $\beta$, galectin-1) or by direct cytotoxic action (granzyme B and perforin release) [3-6]. Phenotypically, Tregs express high levels of FOXP3, cytotoxic T lymphocyte-associated antigen-4 (CTLA-4), certain members of toll-like receptors, CD103 and glucocorticoid-induced TNF receptor family-related gene (GITR) [7-9]. FOXP3 is a more specific marker of Treg, which induces GITR, CD103 and CTLA-4 $[9,10]$. Lymphocyte $\operatorname{Tr} 1$ cells depend on IL-10 for their induction and suppressive action, whereas Th3 cells depend on TGF- $\beta$ for suppressive action. Some Tregs can be induced by IL-35 (iTr35) [10, 11] (Table 1).

Some researchers found deviations in Tregs in peripheral blood of psoriatic patients, which led to hyperactivity of T effectors in vivo [12]. After an effective biologic therapy, an increase in Tregs in blood of psoriatic patients is observed $[13,14]$. However, the literature shows a lot

Address for correspondence: Agnieszka Owczarczyk-Saczonek MD, PhD, Department of Dermatology, Sexually Transmitted Diseases and Clinical Immunology, University of Warmia and Mazury, Al. Wojska Polskiego 30, 10-229 Olsztyn, Poland, phone: +48 896786670 , fax: +48 8967866 75, e-mail: aganek@wp.pl

Received: 21.02.2018, accepted: 3.04.2018. 
Table 1. The characteristics of CD4+ Tregs subtypes

\begin{tabular}{llll}
\hline Cell type & Treg CD4+CD25+ & Tr1 CD4+CD25+ & Th3 CD4+CD25+ \\
\hline Marker & FOXP3+, CD127low, LRRC32/GARP+, & CTLA-4+, LAP+, CD45RBlow, & CTLA-4+, LAP+, CD45RBlow, \\
& CD39+, GITR+, CTLA-4+, LAP+ & FOXP3- & FOXP3+ \\
\hline Mechanism of action & - Cytolysis induction & - Effect on inter-cell contact, & - TGF- $\beta 1$ secretion \\
& - Inhibition of dendritic cells maturation & - IL-10, TGF- $\beta 1$, IFN- $\gamma$ secretion & \\
& - IL-10, IL-35, TGF- $\beta 1$, Galectin-1 & & \\
& secretion & & \\
\hline
\end{tabular}

of contradictory reports. Some authors did not find any differences in the percentage of Tregs between psoriatic and healthy patients $[12,15,16]$.

The discrepancies of the above studies indicate that not the number of Tregs, but their dysfunction may be significant in psoriasis pathogenesis. Tregs isolated from the psoriatic lesions or peripheral blood are functionally deficient in suppressing T effectors cells and are not capable to suppress Th1 in psoriatic patients [17, 18]. In contrast, those isolated from peripheral blood of healthy controls are able to inhibit psoriatic Th1 in vitro [3, 7, 19]. Thus, the dysfunctional Treg cell activity in the blood and psoriatic plaques may eventually result in the reduced restraint and hyperproliferation of psoriatic pathogenic cells in vivo $[17,18]$.

Interleukin-10 has anti-inflammatory properties, inhibiting the production of pro-inflammatory cytokines (IFN- $\gamma$, IL-2, IL-3, TNF- $\alpha, G M-C S F)$. It is produced by Tregs, macrophages, dendritic cells and B lymphocytes. Interleukin-10 inhibits the production of IL-12 by macrophages, which is responsible for Th1 maturation and IFN- $\gamma$ secretion [20]. In psoriasis, a relative deficiency of $\mathrm{IL}-10$ in serum and skin is observed, therefore it seems to be an important factor in psoriatic pathogenesis [21].

Transforming growth factor- $\beta$ is secreted by Treg populations, especially Th3, being an important regulator in immune homeostasis. Moreover, it can limit keratinocyte hyperproliferation in psoriasis [22, 23].

Several studies confirmed an increased TGF- $\beta 1$ expression in lesions and serum in psoriatic patients. TGF- $\beta 1$ levels correlated with the severity of the disease [22]. However, based on clinical data, it is difficult to determine whether increased TGF- $\beta 1$ has a causative function in psoriasis or is a result of the inflammation development. Contrary to plasma levels, the results of TGF- $\beta 1$ in psoriatic lesions are still contradictory.

Another crucial part of analysis on the role of TGF in psoriasis is assessing its isoforms (TGF- $\beta 1$, TGF- $\beta 2$ and TGF- $\beta 3$ ), mediated by specific receptors (TGF $\beta R I, T G F \beta R I$ and TGF $\beta R I I I$ ) [24]. Since TGF- $\beta$ is a potent growth inhibitor for human keratinocytes, the decrease of TGF- $\beta 2$ in the epidermis of psoriatic skin may contribute to epidermal hyperplasia. Moreover, it is confirmed that TGF- $\beta 2$, and not only TGF- $\beta 1$, induces FOXP3 expression in CD4+CD25+ precursors. Therefore, two isoforms of TGF- $\beta$ have been reported to have similar biological ef- fects [24, 25]. Considering much lower binding affinity of TGF- $\beta 2$ to T $\beta$ R-II than TGF- $\beta 1$, TGF-92 may be relatively more potent in inducing FOXP3 expression [25]. Cai et al. revealed that both TGF- $\beta 1$ and TGF- $\beta 2$, and, to a lesser extent, TGF- $\beta 3$ isoforms block the ability of normal but not psoriatic dermal microvascular endothelial cells to bind lymphocytes, as the first stage of inflammation cells migration psoriatic lesions [26]. Therefore, we decided to assess TGF- $\beta 2$ in our study.

Interestingly, the role of IL-35, which belongs to the IL-12 family, has not yet been studied in psoriasis. It is produced mainly by Tregs and downregulates Th17 development, suppressing IL-17 production and promotes IL-10 expression [4, 27-30]. Moreover, some studies suggest that it may reduce the proliferation of CD4+ cells [27]. Zhang et al. found that IL-35 decreased the number of macrophages, and compared with dexamethasone, IL-35 showed long-term therapeutic efficacy. Thus, it can be a new therapeutic strategy for psoriasis and other cutaneous inflammatory diseases [30]. However, the role of IL-35 in psoriasis remains unclear.

\section{Aim}

In this study we evaluated the expression of protective cytokines in serum and Treg markers in lesions and perilesional skin in men with psoriasis in comparison to healthy volunteers.

\section{Material and methods}

\section{Study group}

The study group consisted of 33 Caucasian males with severe plaque psoriasis, without psoriatic arthritis (Table 2). They were recruited according to the Declaration of Helsinki principles. Patients with chronic and acute inflammatory diseases, cancer, cardiovascular diseases, renal and hepatic failure $(n=3)$ have been excluded. Patients were not treated: a wash-out period for topical treatment was 2 weeks, 4 weeks for photo(chemo) therapy and systemic therapy, biologics were not used at all. The control group consisted of 6 males, healthy volunteers without personal or family history of psoriasis. We decided to choose only males, because oestrogen levels can change Treg function and alter the cytokines profile [31]. 
The permission of the Bioethical Committee of the Warmia and Mazury University was obtained (33/2015).

\section{Tissue specimens}

We obtained two 3-mm punch biopsies per patient: one from the centre of the psoriatic plaque (abdominal region), one from non-lesional skin (at least $2 \mathrm{~cm}$ from the target lesion), and one from healthy volunteers (abdominal region), using local anaesthesia (2\% lignocaine). We assessed 66 biopsies from psoriatic patients and 6 from controls.

\section{Enzyme-linked immunosorbent assay (ELISA)}

Determination of cytokines (IL-35, IL-10, TGF- $\beta$ ) was performed using the available kits - for IL-35 with the detection range of 15.6-1000 pg/ml (Wuhan Fine Biological Technology, China), for IL-10: 1.56-50 pg/ml (Diaclone, France) and for TGF- $\beta 1:$ 1.2-600 pg/ml (Demeditec Diagnostic $\mathrm{GmbH}$, Germany), according to the manufacturer's protocol. Validity of the assays was confirmed by parallelism between the standard curves and series of dilutions of randomly chosen plasma samples. The intra-assay coefficient of variation was $<5 \%$. The inter-assay factor of variations was not calculated (all analyses were done in one assay). The sensitivities of the assay were 9.4, 0.98 and $1.9 \mathrm{pg} / \mathrm{ml}$ for IL-35, IL-10 and TGF- $\beta 1$, respectively. We assessed sera from 31 psoriatic patients and 6 from controls.

\section{Immunohistochemistry}

The skin biopsies were dissected into 4- $\mu \mathrm{m}$ thick sections using cryostat CM3050 (Leica, USA) and mounted onto poly-L-lysine-coated glass microscope slides (Menzel-Glaser, Braunschweig, Germany). Frozen sections after reaching room temperature were rinsed three times in $0.01 \mathrm{M} \mathrm{PBS}$ and incubated with $3 \% \mathrm{H}_{2} \mathrm{O}_{2}$ in methanol for $30 \mathrm{~min}$. Next, to decrease nonspecific binding, they were incubated with 5\% bovine albumin serum (Sigma Aldrich, USA) for $60 \mathrm{~min}$ at room temperature. The biopsies were incubated with rabbit polyclonal antibody to IL-27/IL-35, FOXP3, TGF- $\beta 2$ and IL-10 (1 : 50; NovusBio, USA) and mouse monoclonal antibody to CD4, CTLA-4, CD25/IL-2R, CD39/ENTPD1, IL-7R/CD127 and CD3 (1 : 50; NovusBio, USA) diluted in PBS at $4^{\circ} \mathrm{C}$ overnight. The following day, the biopsies were washed three times in PBS and incubated with secondary anti-mouse or anti-rabbit antibodies (1 : 200; Jackson ImmunoResearch, USA) for $60 \mathrm{~min}$. To visualize the immunoreactions, they were immersed in 3.3'-diaminobenzidine (DAB, Dako, USA), next they were dehydrated in ethanol, cleared in xylene and mounted with DPX (Sigma Aldrich, USA). The labelled tissues were photographed using a C-5060 Camera (Olympus, Japan) mounted on a light microscope $(\mathrm{CH} 30 / \mathrm{CH} 40$, Olympus, Japan).
Table 2. Patient demographics

\begin{tabular}{lcc}
\hline Parameter & Psoriatic & Healthy controls \\
\hline$N$ & 33 & 6 \\
\hline Age [years] & $46.45(18-66)$ & $47.3(21-74)$ \\
\hline PASI & $19.58(10.2-45.6)$ & -
\end{tabular}

Values are given as number (median range).

The images were subjected to semiquantitative analysis. Since the parameter defying staining intensity did not differ between the examined stages, the assessed parameter was the immunoreactive area (\%). It was calculated as the ratio of area occupied by the immunopositive cells to the total area occupied by tissue. The level of immunoreactivity was measured with Image software (image processing and analysing in Java, USA). Quantitative analysis was performed using an automatic threshold function to select a range of grey values that were optically identified as positive staining. Before statistical analysis, percentage data were arcsine transformed.

\section{Results}

Concentration of IL-10 and IL-35 in serum are not statistically different in patients with psoriasis and the control group

The serum levels of IL-35 (Figure 1 A) and IL-10 (Figure 1 B) were higher in psoriatic patients, but without statistical significance. Interleukin-10 levels were determined in the range of $1.5 \pm 0.32$ to $4.4 \pm 1.45 \mathrm{pg} / \mathrm{ml}(p=0.56)$ and IL-35 of 13.7 \pm 0.84 to $17.0 \pm 1.38 \mathrm{pg} / \mathrm{ml}(p=0.40)$, respectively. There was no statistically significant relationship between PASI and IL-10 $(r=0.0115)$, IL-35 $(r=-0.0011)$. Correlation analysis showed a statistically significant relationship between IL-10 and IL-35 levels $(r=0.8427)$.

\section{Concentration of serum TGF- $\beta 1$ is increased} in the serum of patients with psoriasis

Concentration of TGF- $\beta 1$ revealed variations (Figure $1 \mathrm{C}$ ) and a higher $(p<0.05)$ level of TGF- $\beta 1$ was noted in the psoriatic patients than in controls $(96.1 \pm 8.62 \mathrm{pg} / \mathrm{ml}$ vs. 34.9 $\pm 3.96 \mathrm{pg} / \mathrm{ml}$ ). There were no statistical correlations between PASI and TGF- $\beta$ levels $(r=0.0156)$.

\section{Immunoreactivity for IL-35, IL-10, TGF- $\beta 2$, CD4, CD3, FOXP3, CD25/IL-2R, CTLA-4, IL-7R/CD127 and CD39/ENTPD1}

The expression of all analysed proteins was noted in three experimental groups (lesional, perilesional and healthy skin). The level of immunoreactive IL-35, IL-10, TGF- $\beta 2$, CD4, CD3, FOXP3 and CD25/IL-2R proteins varied in different experimental groups $(p<0.05)$. The level of IL-35 was the lowest in the skin biopsies from psoriatic lesions ( $p<0.05)$ compared to healthy and perilesional skin (Figure 2 A). The level of CD4 (Figure 2 B), IL-10 (Fig- 

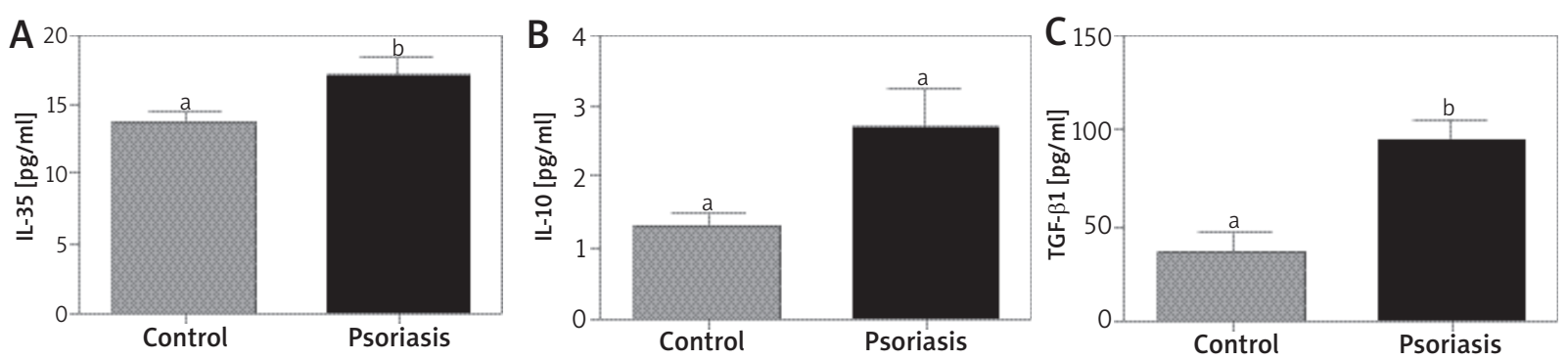

Figure 1. Concentration of IL-35 (A), IL-10 (B) and TGF- $\beta 1(C)$ in the control group $(n=6)$ and patients with psoriasis $(n=33)$. Bars with different letters are significantly different $(p<0.05)$

ure 2 C) and TGF- $\beta 2$ (Figure 2 D) were higher $(p<0.05)$ in perilesional skin than in lesions. TGF- $\beta 2$ expression was decreased in lesional and perilesional skin compared to controls (Figure $2 \mathrm{D}, p<0.05$ ).

The variance of CD25/IL2R (Figure $2 \mathrm{G}$ ) was characterized by the increase in healthy skin $(p<0.05)$ compared to perilesional and lesional $(p<0.05)$ (Figure $2 \mathrm{G}$ ) with no differences between them. On the contrary, the elevated level of FOXP3 expression was noted in lesions only (Figure $2 \mathrm{~F}$ ). There was no difference between experimental groups in CTLA-4 (Figure 2 H), IL7R/CD127 (Figure 2 I) and CD39/ENTPD1 (Figure $2 \mathrm{~J}$ ) protein expression.

There was a close correlation between IL-10 and IL-35 both in the controls $(r=0.9099)$, perilesional $(r=$ $0.5236)$ and lesional skin $(r=0.4514)$. TGF- $\beta 2$ expression was higher $(p<0.05)$ in the perilesional epidermal and dermal layer than in psoriatic lesions (Figure $2 \mathrm{D}$ ).

Expressions of CD25, CD3, FoxP3, CD39, IL-10, IL35 and CTLA-4 were higher $(p<0.05)$ in the dermis, both in the papillary and reticular layer. There were no significant differences in the expression of TGF- $\beta 2$ and IL-7R between skin and epidermis (Table 3).

\section{Discussion}

We observed that the levels of IL-10 and IL-35 in serum are higher, but not statistically different from patients with psoriasis and the control group. Similarly to our results, Borska et al. reported that IL-10 levels were significantly higher in psoriatic patients, and decreased after the treatment [32]. The same results were obtained by Deeva et al. without any correlation between PASI and IL-10 in mild-to-moderate psoriatic patients [33].

In contrast to our results, other authors found a significant decrease in serum IL-10 levels in psoriatic patients compared to healthy controls [34, 35]. Verghese et al. found that IL-10 levels were higher in controls than in patients without statistical significance [36]. Similarly, Takahashi et al. detected decreased serum levels of IL-10 in psoriasis which negatively correlated with PASI [37]. However meta-analysis of 6 researches showed a small, positive but not statistically significant difference between psoriatic patients and controls. It did not depend on age, sex, PASI or psoriasis type [38]. These controversial results do not confirm IL-10 as a reliable biomarker for psoriasis.

Despite the significant anti-inflammatory effect of IL-10 and reports of its effectiveness in psoriasis therapy, the results of serum psoriasis studies are not conclusive. However, the genetic polymorphism of IL-10 may affect the expression of this cytokine.

In the literature, there are a few reports about IL-35 in psoriasis. Cardoso et al. did not find any differences between the level of IL-35 in the serum of Brazilian psoriatic patients and healthy controls [27]. Li et al. also showed that serum levels of IL-35 were higher in patients with psoriatic arthritis (especially in erosive phases) just as in healthy controls. This result suggested that IL-35 was closely related to arthritis [29].

We assessed significantly higher levels of serum TGF- $\beta 1$ of patients with psoriasis than controls as some other researchers [22, 39-42]. Kallimanis et al. observed a decrease in TGF- $\beta 1$ together with a decrease in PASI after biological treatment. Hence, TGF- $\beta 1$ levels seem to be sensitive to changes in disease severity [39].

On the other hand, Cardoso et al. did not find statistical significances for TGF- $\beta$ in serum of Brazilian psoriasis patients and healthy participants [27]. Similarly, Zaher et al. observed that the mean serum levels of TGF- $\beta 1$ in psoriatic patients were higher than controls but statistically non-significant, although correlating with PASI [42].

Probably the increased TGF- $\beta 1$ could be a result of activated endothelial cells, fibroblasts, or inflammatory cells which can produce TGF- $\beta 1$ in psoriasis [22].

Our immunohistochemical investigations suggested a low cutaneous IL-10 expression. Similar results were found by quantification of IL-10 protein in blister fluids [21, 43, 44]. However, conflicting data have been published. Overexpression has been reported by some authors, but the groups of patients were too small [45, 46].

There is no study evaluating IL-35 expression in psoriasis. We found a close correlation between IL-10 and IL-35 both in the controls, perilesional and lesional skin. It suggested an anti-inflammatory role of IL-35 in psoriasis.

We found a higher TGF- $\beta 2$ expression in the perilesional epidermal and dermal layer than in psori- 

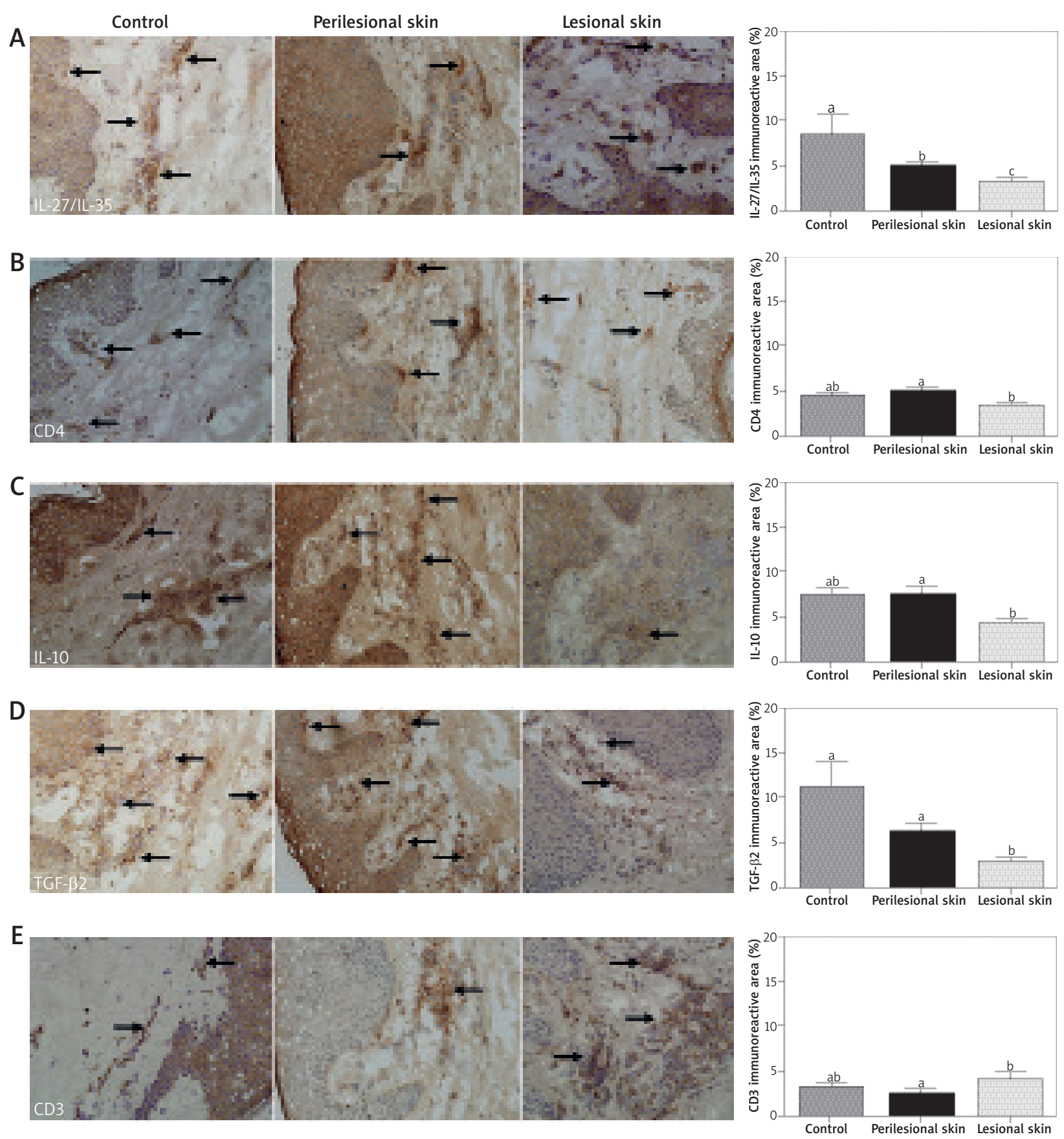

Figure 2. The immunoreactive area of IL-27/IL-35 (A), CD4 (B), IL-10 (C), TGF- $\beta 2$ (D), CD3 (E), FOXP3 (F), CD25/IL-2R (G), CTLA-4 (H), IL-7R/CD127 (I) and CD39/ENTPD1 (J) proteins in the sections from healthy skin $(n=8)$, perilesional skin $(n=$ 33) and skin with psoriatic lesions $(n=33)$. Bars with different letters are significantly different $(p<0.05)$. The proteins are marked in brown (3,3'-diaminobenzidine-DAB). Nuclei were stained with hematoxylin (violet) (magnification 400x)

atic lesions. Wataya-Kaneda et al. found a decreased amount of TGF- $\beta 2$ in the psoriatic epidermis, which contributes to epidermal hyperplasia. The intensity of immunoreactivity has the tendency to decrease in the lower epidermis rather than in the upper epidermis of the transitional lesion [47]. Flisiak et al. revealed no correlation between PASI and TGF- $\beta 1$, TGF- $\beta 2$ in psori- atic lesions with a significant correlation only between TGF- $\beta 1$ concentration in scales/size and the disease duration [48]. The study of Yu et al. demonstrated a significant activity of TGF- $\beta 3$ and TGF $\beta$ RII mRNA only in non-lesional psoriatic skin but there was no significant difference in the expression of TGF- $\beta 1$ and TGF- $\beta 2$ [49]. 

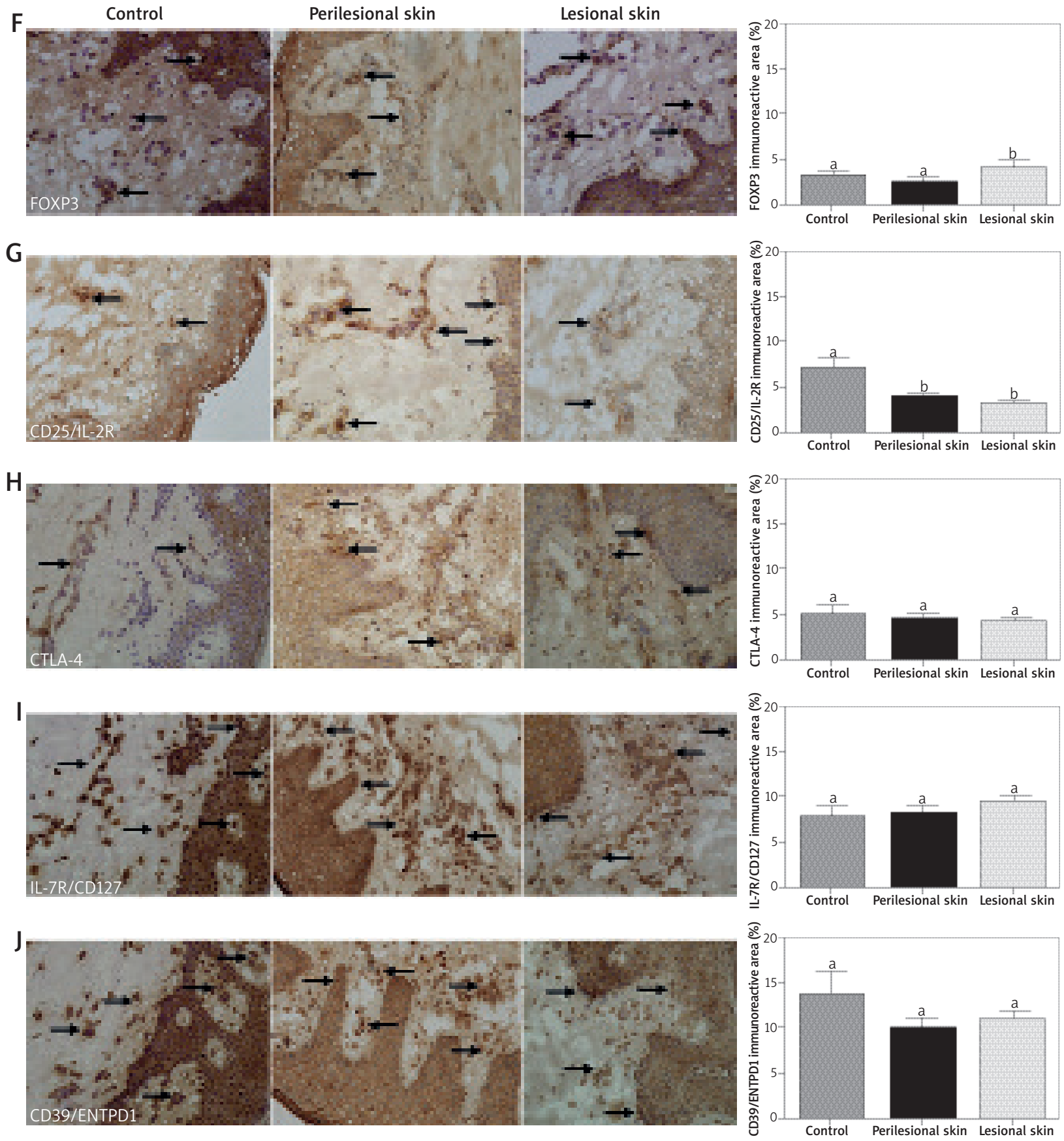

Figure 2. Cont. The immunoreactive area of IL-27/IL-35 (A), CD4 (B), IL-10 (C), TGF- $\beta 2$ (D), CD3 (E), FOXP3 (F), CD25/IL-2R (G), CTLA-4 (H), IL-7R/CD127 (I) and CD39/ENTPD1 (J) proteins in the sections from healthy skin $(n=8)$, perilesional skin $(n=33)$ and skin with psoriatic lesions $(n=33)$. Bars with different letters are significantly different $(p<0.05)$. The proteins are marked in brown (3,3'-diaminobenzidine - DAB). Nuclei were stained with hematoxylin (violet) (magnification 400x)

Interestingly, our results revealed a reduced expression of IL-10, IL-35 and TGF- $\beta 1$ in psoriatic lesions compared to perilesional skin. This may indicate their involvement in inhibiting inflammation in psoriasis and their amount decreases due to their wear off. It suggests an anti-inflammatory role of IL-35 in psoriasis, although it belongs to the proinflammatory group of the IL-12 family.
We confirmed a higher concentration of CD3 (co-receptor of cytotoxic T-cells and Th) in psoriatic lesions compared to perilesional skin. Similar results were obtained by other authors [50-52]. This is a typical phenomenon associated with the involvement in psoriatic inflammation of Th1, Th17 and Th22. Interestingly, in our study, the concentrations of CD4 proteins were higher in perilesional skin than in lesions. 
Table 3. Expression of Treg markers and protective cytokines in the healthy, lesional and perilesional psoriatic skin immunohistochemistry

\begin{tabular}{|c|c|c|c|c|c|c|}
\hline \multirow[t]{3}{*}{ Parameter } & & \multirow[t]{3}{*}{ Healthy skin } & \multicolumn{4}{|c|}{ Psoriatic skin } \\
\hline & & & \multicolumn{2}{|c|}{ Perilesional skin } & \multicolumn{2}{|c|}{ Psoriatic lesions } \\
\hline & & & Epidermis & Dermis & Epidermis & Dermis \\
\hline \multirow[t]{7}{*}{ Treg markers } & CD4 & + & + & + & + & + \\
\hline & CD-25 (2R) & + & + & ++ & + & + \\
\hline & CD3 & ++ & + & ++ & + & ++ \\
\hline & FOXP3 & + & + & ++ & + & ++ \\
\hline & CD39 & +++ & ++ & +++ & ++ & +++ \\
\hline & CTLA-4 & ++ & + & ++ & + & + \\
\hline & IL7R/CD127 & +++ & +++ & +++ & +++ & +++ \\
\hline \multirow{3}{*}{$\begin{array}{l}\text { Anti- } \\
\text { inflammatory } \\
\text { cytokines }\end{array}$} & IL-10 & ++ & + & ++ & + & ++ \\
\hline & TGF- $\beta$ & + & + & + & + & + \\
\hline & IL-35 & ++ & + & ++ & + & ++ \\
\hline
\end{tabular}

$+++>50 \%$ cells are positive, ++ minority of cells are positive, + a few cells are positive.

To evaluate Treg activity, an assessment of CD25 and FOXP3 expression was made. However, the intracellular location of FOXP3 limits its usefulness in studying and purifying this subpopulation. Cell surface CD25 expression is often used as a marker for CD4+Tregs, but CD25 is somewhat nonspecific [53]. There are only a few studies devoted to the evaluation of other markers of Treg in the literature, including CTLA-4, chemokine receptors (CCR4 and CCR8), selectins (CD62L), integrins (CD103), and CD127. In vivo, putative mechanisms include the release of soluble factors IL-10 and TGF- $\beta[53,54]$. Their role is not fully understood in psoriasis. We made an assessment of some of the most important ones, bearing in mind the heterogeneity of the Treg group.

Our results were similar to the other studies. Psoriatic lesions exhibit an increased FOXP3+ expression compared to the healthy skin $[18,19,55,56]$. There are an increased number of FOXP3+Treg in psoriatic lesions compared to controls and they were located predominantly in the papillary and upper reticular layers of the dermis [56]. We observed an elevated expression of FOXP3 in lesional dermis compared to perilesional skin. However, in psoriatic patients, Tregs readily turn into IL-17-expressing cells, which potentially perpetuate the inflammatory process that characterizes the disease [57]. Bovenschen et al. proved that psoriatic Tregs exhibit a propensity to alter levels of the main regulator FOXP3 and upregulating the expression of ROR $\gamma$ t by the addition of proinflammatory cytokines characteristic for psoriatic lesions (IL-2, IL-15, IL-1 $\beta$, and IL-23), together with a decrease in FOXP3 and an increase in IL-17-producing Treg. Moreover, the conversion from Treg to IL-17/Treg is a continuum of converting cells which exists, as evidenced by FOXP3+ ROR $y \mathrm{t}+$ co-expression and a gradual loss of FOXP3 [19]. In this way we can explain the lack of differences in the expression of other Treg-specific molecules in the study groups. What is more, the expression of CD3, characteristic of T-cell glycoprotein, has been found higher in the dermis.

However Keijsers et al. found a significant increase in CD3+, CD4+ and FOXP3+cells in the transition from the symptomless to lesional skin. They were higher in the distant uninvolved skin than in the perilesional, lesional skin and even higher than in healthy skin. They obtained a high FOXP3/CD4 ratio in the symptomless skin of psoriatic patients [58]. Yan et al. detected an increase fraction in the psoriatic lesions irrespective of the severity of disease as compared to normal skin, but a decrease in FOXP3+cells from acute psoriatic biopsies. They suggested that FOXP3+Treg plays a crucial role in exacerbation of psoriasis not in a stable phase [56].

It is difficult to explain why we obtained a higher expression of $C D 4+$ in perilesional skin than in psoriatic lesions. Moreover, CD4 is present not only on Tregs but is characteristic of Th1 and Th17. We expected to observe increasing CD4 in the lesional skin.

CD25(IL-2R) is present on activated $T$ and $B$ cells and used as a marker to identify CD4+FOXP3+Treg [59]. Most psoriasis-associated $T$ cells are CD3+CD2+CD45RO+CLA+ with a subset having activation markers CD25, HLA-DR, and CD27 [60]. Therefore we reported a higher $(p<0.05)$ concentration of CD25/IL2R in control skin compared to psoriatic skin without any differences between the perilesional and lesional skin.

Duncan et al. demonstrated a significant correlation between the number of IL-2R+(CD25+) cells in lesional skin and PASI [61]. Similarly, Ferenczi et al. detected high numbers of IL-2R+T cells in lesional skin (epidermal and dermal layer) with an early activation of associated CD25 ( $\alpha$ chain) molecule and with CD122 ( $\beta$ chain) [62]. 
CD39 expression has been described on FOXP3+Treg. It is the rate-limiting enzyme in the hydrolysis of extracellular ATP and ADP into AMP. Binding of adenosine leads to a rise of intracellular CAMP levels, which subsequently suppresses T effectors function such as proliferation and CD25 up-regulation [63]. The role of CD39+FOXP3+Treg has been investigated in multiple sclerosis: circulating CD39+FOXP3+Treg were diminished and impaired while CD39+FOXP3+Treg healthy individuals were suppressed by Th17 [64].

We did not find any differences in CD39 expression, contrary to others. They were scattered throughout the epidermis and dermis [19, 31, 56]. Zhang et al. assessed the proportions of CD39/CD73 expressing FOXP3+Treg in different types of psoriatic lesions and controls. In normal skin, CD39+cells were present and localized predominantly in the dermis, especially in the papillary and upper reticular layers of the dermis, and minimally in the epidermis. The proportion of cells in both CD39+ and FOXP3+ were significantly lower in pustular and erythrodermic psoriasis than in plaque psoriasis [30]. Similar results were reported by Bovenschen et al. and Yan et al. $[19,56]$. However, they did not achieve a significant reduction in plaque psoriasis expression compared to pustular and erythrodermic psoriasis.

Tregs are characterized by low CD127(IL-7R $\alpha$ ) expression compared to conventional T cells. In our study, the expression of IL-7R/CD127 did not differ in analysed groups. Simonetta et al. investigated high CD127 expression on Treg in vitro and in vivo in contact dermatitis models. The opposite regulation of CD127 on Tregs and T effectors during activation led to equivalent CD127 expression on these two subsets. It is probably an additional mechanism for the functional impact of IL-17 on Treg suppression, which has been shown to rescue effector cells from Treg-mediated apoptosis [65].

CTLA4 is a critical negative regulator of T-cell activation and induces an inhibitory effect on B-cell inactivity, with a consequent decrease in autoantibody formation, decrease in macrophage activation, and reduction in proinflammatory cytokines $[66,67]$. We observed higher $(p<0.05)$ CTLA-4 expression in psoriatic lesions, especially in the papillary layer.

The problem of our study is that the $T$ effectors may exhibit transiently expressing CD25 and/or CTLA-4 in epidermis and dermis $[18,68]$. Therefore Sugiyama et al. showed that epidermal and dermal cell suspensions rested for $48 \mathrm{~h}$ before flow cytometric analysis, because in CD4+CD25+Treg CTLA-4 is expressed up to 7 days after activation. They calculated the ratio of CD4+CD25+Treg vs. CD4+CD25- T effectors in psoriatic lesions to be $1: 6$ in dermis and $1: 2$ in epidermis [18].

Limitations of our research are a low number of patients and ambiguous identification of Tregs. Immunohistochemistry cannot provide convincing evidence for Treg identification.

\section{Conclusions}

The differences between the levels of protective cytokines and expression of Treg markers in perilesional skin and psoriatic lesions can be a proof of major disorders regarding the mechanisms of immune tolerance and might explain the inflammation development in psoriasis. Heterogeneous results of researches still suggest the need for further research on this topic.

\section{Acknowledgments}

This work was financed by the grant awarded by the University of Warmia and Mazury 33/2015.

\section{Conflict of interest}

The authors declare no conflict of interest.

\section{References}

1. Deng Y, Chang C, Lu Q. The inflammatory response in psoriasis: a comprehensive review. Clin Rev Allergy Immunol 2016; 50: 377-89

2. Karamehic J, Zecevic L, Resic H, et al. Immunophenotype lymphocyte of peripheral blood in patients with psoriasis. Arch Med 2014; 68: 236-8.

3. Birch KE, Vukmanovic-Stejic M, Reed JR, et al. The role of regulatory $T$ cells in cutaneous disorders. In: Regulatory T Cells in Inflammation. Leonie S, Arne N, Marca HM (eds.) Springer Science \& Business Media 2006.

4. Buckner JH. Mechanisms of impaired regulation by CD4(+) CD25(+)FOXP3(+) regulatory T cells in human autoimmune diseases. Nat Rev Immunol 2010; 10: 849-59.

5. Kondelková K, Vokurková D, Krejsek J, et al. The number of immunoregulatory $T$ cells is increased in patients with psoriasis after Goeckerman therapy. Acta Medica (Hradec Kralove) 2012; 55: 91-5.

6. Vignali DA. How regulatory T cells work. Nat Rev Immunol 2008; 8: 523-32.

7. Kagen MH, McCormick TS, Cooper KD. Regulatory T cells in psoriasis. Ernst Schering Res Found Workshop 2006; 56 : 193-209.

8. Liu H, Leung BP. CD4 ${ }^{+} \mathrm{CD} 25^{+}$regulatory T cells in health and disease. Clin Exp Pharmacol Physiol 2006; 33: 519-24.

9. Yun WJ, Lee DW, Chang SE, et al. Role of CD4+CD25 $5^{\text {high+ }} \mathrm{FOXP3}^{+}$ regulatory T cells in psoriasis. Ann Dermatol 2010; 22: 397403.

10. Broere F, Apasov SG, Sitkovsky MV, et al. T cell subsets and T cell-mediated immunity. In: Principles of Immunopharmacology. Nijkamp FP, Parnham MJ. Springer Basel AG 2011; 15-7.

11. Zeng $H$, Zhang $R$, Jin B, et al. Type 1 regulatory T cells: a new mechanism of peripheral immune tolerance. Cell Mol Immunol 2015; 12: 566-71.

12. Furuhashi T, Saito C, Torii K, et al. Photo(chemo)therapy reduces circulating Th17 cells and restores circulating regulatory T cells in psoriasis. PLoS One 2013; 8: e54895.

13. Quaglino P, Bergallo M, Ponti R, et al. Th1, Th2, Th17 and regulatory $T$ cell pattern in psoriatic patients: modulation of cytokines and gene targets induced by etanercept treatment and correlation with clinical response. Dermatology 2011; 223: 57-67. 
14. Richetta AG, Mattozzi C, Salvi M, et al. CD4+ CD25+ T-regulatory cells in psoriasis. Correlation between their numbers and biologics-induced clinical improvement. Eur J Dermatol 2011; 21: 344-8.

15. Saito C, Maeda A, Morita A. Bath-PUVA therapy induces circulating regulatory $T$ cells in patients with psoriasis. J Dermatol Sci 2009; 53: 231-3.

16. Zhang L, Yang XQ, Cheng J, et al. Increased Th17 cells are accompanied by FoxP3(+) Treg cell accumulation and correlated with psoriasis disease severity. Clin Immunol 2010; 135: 108-7.

17. Karczewski J, Dobrowolska A, Rychlewska-Hańczewska A, et al. New insights into the role of T cells in pathogenesis of psoriasis and psoriatic arthritis. Autoimmunity 2016; 49: 435-50.

18. Sugiyama H, Gyulai R, Toichi E, et al. Dysfunctional blood and target tissue CD4+CD25high regulatory T cells in psoriasis: mechanism underlying unrestrained pathogenic effector T cell proliferation. J Immunol 2005; 174: 164-73.

19. Bovenschen HJ, van Vlijmen-Willems IM, van de Kerkhof PC, et al. Identification of lesional CD4+CD25+Foxp3+ regulatory T cells in psoriasis. Dermatology 2006; 213: 111-7.

20. Döcke WD, Asadullah K, Belbe G, et al. Comprehensive biomarker monitoring in cytokine therapy: heterogeneous, time-dependent, and persisting immune effects of interleukin-10 application in psoriasis. J Leukoc Biol 2009; 85: 582-93.

21. Asadullah K, Sabat R, Friedrich M, et al. Interleukin-10: an important immunoregulatory cytokine with major impact on psoriasis. Curr Drug Targets Inflamm Allergy 2004; 3: 185-92.

22. Flisiak I, Zaniewski P, Chodynicka B. Plasma TGF-beta1, TIMP-1, MMP-1 and IL-18 as a combined biomarker of psoriasis activity. Biomarkers 2008; 13: 549-56.

23. Litvinov IV, Bizet AA, Binamer Y, et al. CD109 release from the cell surface in human keratinocytes regulates TGF-beta receptor expression, TGF beta signaling and STAT3 activation: relevance to psoriasis. Exp Dermatol 2011; 20: 627-32.

24. Derynck R, Zhang YE. SMAD-dependent and SMAD-independent pathways in TGF-beta family signalling. Nature 2003; 425: 577-84.

25. Fu S, Zhang N, Yopp AC, et al. TGF-beta induces Foxp3 + Tregulatory cells from CD4+CD25- precursors. Am J Transplant 2004; 4: 1614-27.

26. Cai JP, Falanga V, Taylor JR, et al. Transforming growth factorbeta receptor binding and function are decreased in psoriatic dermal endothelium. J Invest Dermatol 1996; 106: 225-31.

27. Cardoso PR, Lima EV, Lima MM, et al. Clinical and cytokine profile evaluation in Northeast Brazilian psoriasis plaquetype patients. Eur Cytokine Netw 2016; 27: 1-5.

28. Collison LW, Vignali DA. Interleukin-35: odd one out or part of the family? Immunol Rev 2008; 226: 248-62.

29. Li Y, Wang Y, Liu Y, et al. The possible role of the novel cytokines IL-35 and IL-37 in Inflammatory bowel disease. Med Inflam 2014; 2014: 136329.

30. Zhang J, Lin Y, Li C, et al. IL-35 Decelerates the inflammatory process by regulating inflammatory cytokine secretion and M1/M2 macrophage ratio in psoriasis. J Immunol 2016; 197: 2131-44.

31. Zeng QM, Liu DC, Zhang XC, et al. Estrogen deficiency inducing shifted cytokines profile in bone marrow stromal cells inhibits Treg cells function in OVX mice. Cell Mol Biol 2015; 61: 64-8.

32. Borska L, Andrys C, Krejsek J, et al. Serum levels of the proinflammatory cytokine interleukin-12 and the anti-inflam- matory cytokine interleukin-10 in patients with psoriasis treated by the Goeckerman regimen. Int J Dermatol 2008; 47: 800-5.

33. Deeva I, Mariani S, De Luca C, et al. Wide-spectrum profile of inflammatory mediators in the plasma and scales of patients with psoriatic disease. Cytokine 2010; 49: 163-70.

34. Jacob SE, Nassiri M, Kerdel FA, et al. Simultaneous measurement of multiple Th1 and Th2 serum cytokines in psoriasis and correlation with disease severity. Mediators Inflamm 2003; 12: 309-13.

35. Roussaki-Schulze AV, Kouskoukis C, Petinaki E, et al. Evaluation of cytokine serum levels in patients with plaque-type psoriasis. Int J Clin Pharmacol Res 2005; 25: 169-73.

36. Verghese B, Bhatnagar S, Tanwar R, et al. Serum cytokine profile in psoriasis-a case-control study in a tertiary care hospital from northern India. Indian J Clin Biochem 2011; 26: 373-7.

37. Takahashi H, Tsuji H, Hashimoto Y, et al. Serum cytokines and growth factor levels in Japanese patients with psoriasis. Clin Exp Dermatol 2010; 35: 645-9.

38. Dowlatshahi EA, van der Voort EA, Arends LR, et al. Markers of systemic inflammation in psoriasis: a systematic review and meta-analysis. Br J Dermatol 2013; 169: 266-82.

39. Kallimanis PG, Xenos K, Markantonis SL, et al. Serum levels of transforming growth factor-beta1 in patients with mild psoriasis vulgaris and effect of treatment with biological drugs. Clin Exp Dermatol 2009; 34: 582-6.

40. Meki AR, Al-Shobaili H. Serum vascular endothelial growth factor, transforming growth factor beta1, and nitric oxide levels in patients with psoriasis vulgaris: their correlation to disease severity. J Clin Lab Anal 2014; 28: 496-501.

41. Nockowski P, Szepietowski J.C, Ziarkiewicz M, et al. Serum concentrations of transforming growth factor beta 1 in patients with psoriasis vulgaris. Acta Dermatovenerol Croat 2004; 12: 2-6.

42. Zaher H, Shaker OG, EL-Komy MH, et al. Serum and tissue expression of transforming growth factor beta 1 in psoriasis. J Eur Acad Dermatol Venereol 2009; 23: 406-9.

43. Al-Robaee AA, Al-Zolibani AA, Al-Shobili HA, et al. IL-10 implications in psoriasis. Int J Health Sci 2008; 2: 53-8.

44. Mussi A, Bonifati C, Carducci M, et al. IL-10 levels are decreased in psoriatic lesional skin as compared to the psoriatic lesion-free and normal skin suction blister fluids. J Biol Regul Homeost Agents 1994; 8: 117-20.

45. Lemster BH, Carroll PB, Rilo HR, et al. 1995. IL-8/IL-8 receptor expression in psoriasis and the response to systemic tacrolimus (FK506) therapy. Clin Exp Immunol 1995; 99: 148-54.

46. Olaniran AK, Baker BS, Paige DG, et al. Cytokine expression in psoriatic skin lesions during PUVA therapy. Arch Dermatol Res 1996; 288: 421-5.

47. Wataya-Kaneda M, Hashimoto K, Kato M, et al Differential localization of TGF-beta-precursor isotypes in psoriatic human skin. J Dermatol Sci 1996; 11: 183-8.

48. Flisiak I, Chodynicka B, Porebski P, et al. Association between psoriasis severity and transforming growth factor beta1 and beta 2 in plasma and scales from psoriatic lesions. Cytokine 2002; 19: 121-5.

49. Yu H, Mrowietz U, Seifert O. Downregulation of SMAD2, 4 and 6 mRNA and TGFbeta receptor I mRNA in lesional and non-lesional psoriatic skin. Acta Derm Venereol 2009; 89: 351-6.

50. Guttman-Yassky E, Nograles KE, Krueger JG. Contrasting pathogenesis of atopic and psoriasis-part I: clinical and pathologic concepts. J Allergy Clin Immunol 2011; 127: 1110-8. 
51. Khairutdinov VR, Mikhailichenko AF, Belousova IE, et al. The role of intradermal proliferation of T-cells in the pathogenesis of psoriasis. An Bras Dermatol 2017; 92: 41-4.

52. Noda S, Krueger JG, Guttman-Yassky E. The translational revolution and use of biologics in patients with inflammatory skin diseases. J Allergy Clin Immunol 2015; 135: 324-36.

53. Deaglio S, Dwyer KM, Gao W, et al Adenosine generation catalyzed by CD39 and CD73 expressed on regulatory T cells mediates immune suppression. J Exp Med 2007; 204: 1257-65.

54. Chen ML, Pittet MJ, Gorelik L, et al. Regulatory T cells suppress tumor-specific CD8 T cell cytotoxicity through TGF-beta signals in vivo. Proc Natl Acad Sci USA 2005; 102: 419-24.

55. de Boer OJ, van der Loos CM, Teeling P, et al. Immunohistochemical analysis of regulatory $T$ cell markers Foxp3 and GITR on CD4+CD25+ T cells in normal skin and inflammatory dermatoses. J Histochem Cytochem 2007; 55: 891-8.

56. Yan KX, Fang X, Han L. Foxp3+ regulatory T cells and related cytokines differentially expressed in plaque vs. guttate psoriasis vulgaris. Br J Dermatol 2010; 163: 48-56.

57. Soler DC, McCormick TS. The dark side of regulatory T cells in psoriasis. I Invest Dermatol 2011; 131: 1785-6.

58. Keijsers RR, van der Velden HM, van Erp PE, et al. Balance of Treg vs. T-helper cells in the transition from symptomless to lesional psoriatic skin. Br J Dermatol 2013; 168: 1294-302.

59. Triplett, Curti BD, Bonafede PR, et al. Defining a functionally distinct subset of human memory CD4+ T cells that are CD25POS and FOXP3NEG. Eur I Immunol 2012; 42: 1893-905.

60. Lowes MA, Suárez-Farińas M, Krueger JG. Immunology of psoriasis. Annu Rev Immunol 2014; 32: 227-55.

61. Duncan JI, Horrocks C, Ormerod AD, et al. Soluble IL-2 receptor and CD25 cells in psoriasis: effects of cyclosporin A and PUVA therapy. Clin Exp Immunol 1991; 85: 293-6.

62. Ferenczi K, Burack L, Pope M, et al. CD69, HLA-DR and the IL-2R identify persistently activated T cells in psoriasis vulgaris lesional skin: blood and skin comparisons by flow cytometry. J Autoimmun 2000; 14: 63-78.

63. Herrath J, Chemin K, Albrecht I, et al. Surface expression of CD39 identifies an enriched Treg-cell subset in the rheumatic joint, which does not suppress IL-17A secretion. Eur J Immunol 2014; 44: 2979-89.

64. Fletcher JM, Lonergan R, Costelloe L, et al. CD39+Foxp3+ regulatory T cells suppress pathogenic Th17 cells and are impaired in multiple sclerosis. I Immunol 2009; 183: 7602-10.

65. Simonetta F, Chiali A, Cordier C, et al. Increased CD127 expression on activated FOXP3+CD4+ regulatory T cells. Eur J Immunol 2010; 40: 2528-38.

66. Liang J, Zhang S, Luo Q, et al. Lack of association between cytotoxic T-lymphocyte antigen-4+49A/G polymorphism and psoriasis and vitiligo: a meta-analysis of case-control studies. Gene 2015; 568: 196-202.

67. Schiotis R, Buzoianu A, Mureșanu D, et al. New pharmacological strategies in rheumatic diseases. J Med Life 2016; 9: 227-34.

68. Dieckmann D, Plottner H, Berchtold S, et al. Ex vivo isolation and characterization of CD4(+)CD25(+) T cells with regulatory properties from human blood. J Exp Med 2001; 193: 1303-10. 